\title{
Development of new approaches to moisture content measurement for building materials
}

\author{
M. C. Phillipson ${ }^{1}$, P. H. Baker ${ }^{1}$, A. McNaughtan ${ }^{1}$, \\ M. Davies ${ }^{2} \&$ Z. $\mathrm{Ye}^{2}$ \\ ${ }^{1}$ Glasgow Caledonian University, Glasgow, UK \\ ${ }^{2}$ University College London, London, UK
}

\begin{abstract}
The measurement of moisture in building materials has been of importance to building professionals for many years to aid the diagnosis of the nature and cause of building defects. Measurements of moisture content of the building fabric are often carried out on elements where there is uncertainty about the material composition, uncertainty about the dimensions of individual components, and where there is an existing problem there can be some degradation of the materials themselves. Accurate measurements of the moisture within such walls present many challenges.

Research has been undertaken to evaluate the practical application of three relatively new techniques in building science: dual probe heat pulse method; time domain reflectometry; and more sophisticated electrical approaches. Although these techniques have been used in different sciences, the application to actual buildings can present a challenge. This paper details the development of these techniques from theoretical concept through to a practical technique successfully applied for real measurements. The techniques have been calibrated against X-ray absorption measurements using materials of well-defined properties such as sandstone and autoclaved aerated concrete. This calibration allows absolute measurements of moisture content to be made. Finally the paper explores the practicalities of using these approaches for in-situ measurements and identifies particular opportunities and limitations for future application.
\end{abstract}

Keywords: moisture content, practical application, time domain reflectometry, heat pulse measurements, electrical measurements. 


\section{Introduction}

The diagnosis and remediation of many building defects requires measurement of moisture in building materials. Moisture content measurements of building fabric are often carried out on elements where there is uncertainty about the material composition, uncertainty about the dimensions of individual components, and where there is an existing problem there can be some degradation of the materials themselves. Accurate measurements of the moisture within such walls can present many challenges.

Research has been undertaken to evaluate the practical application of three relatively new techniques: dual probe heat pulse method; time domain reflectometry; and more sophisticated electrical approaches. Although these techniques have been used in different sciences, the application to actual buildings can present a challenge. This paper reports the relative merits of the different techniques and comments on their scope for practical application.

\section{Practical limitations of site measurements}

Site measurements techniques should give consistent results and be relatively simple to use. Although the main commercial techniques used for site measurements have changed relatively little in the last few decades, some research tools have been examined for potential site applications. For example, the development of portable NMR systems [1] has been investigated. As with commercial techniques, there are associate limitations with research techniques applied to site measurements. These limitations are associated with the building itself and with the measurement method. These are described in more detail below.

\subsection{Building limitations}

It is rare for the exact design and material composition of a building to be known and its properties catalogued. Although an experienced surveyor may make judgements about the construction of a building, there will often be variation. This is particularly true for old stone buildings.

Within the laboratory environment, equipment can be calibrated for measurements of specific materials, achieving high measurement accuracy. Preconditioning of samples within the laboratory can achieve defined initial moisture contents and identifiable moisture histories. Samples can be produced of known homogeneity and dimensions, whereas on site the nature of the building fabric is typically less accurately described. Uncertainty in the materials used for the construction gives increased errors associated with the measurement. There is also the potential for materials to be present (for example salts), which may not be visible, and which interfere with some types of measurement. The accuracy that can be realised with site measurements will be significantly less than that which can be achieved using the same technique in the laboratory. 


\subsection{Technique limitations}

Each individual technique has particular strengths and weaknesses. The following is an indication of the nature of some of the weaknesses with respect to site application:

- $\quad$ Duration: The length of time taken to make a measurement may make the technique unsuitable for rapid assessment of a large area, or for long term monitoring of a building.

- Destructive Nature: Holes are required for some techniques to either remove material, or insert a probe. This can be unsuitable for important historic materials.

- Susceptibility to Interference: The presence of metal components or salts close to the measurement location can strongly affect some techniques.

- $\quad$ Contact Issues: The quality of contact between a probe and the material being measured can be very important for some techniques. Poor surfaces, treated surfaces, or difficulties installing probes may lead to inaccurate measurements.

\section{Techniques investigated}

Techniques used in other fields of science are being developed for moisture measurement in buildings. Two approaches have been identified, which may have significant development potential for building applications: The Dual Probe Heat Pulse Method [2], and Time Domain Reflectometry [3] (TDR). A third approach being investigated is the development of a more sophisticated instrument based on the measurement of electrical resistance [4]. The theory and performance of the techniques is described in detail elsewhere [2, 3]. These techniques have been successfully applied in laboratory conditions by the authors.

\subsection{Time domain reflectometry}

TDR measures the transmission time of a signal along parallel stainless steel waveguides and its subsequent re-reflection to give a measurement of the relative permittivity $\left(\varepsilon_{\mathrm{r}}\right)$ of the surrounding media, which is directly correlated with moisture content. Equation (1) relates the signal transmission time ( $\mathrm{t}$ ), with the relative permittivity, the length $(\mathrm{L})$ of the waveguides and the velocity of light (c) in a vacuum.

$$
t=\frac{2 L \sqrt{\varepsilon_{r}}}{c}
$$

Each measurement takes little time and has no significant impact on the substrate so TDR can monitor changes of moisture content over a long period. 
However, the transmission time for a signal and its reflection is extremely short so high precision quality electronics are required to minimise systematic error.

Probes can be calibrated with liquids of defined dielectric properties to ensure that accurate measurements of the relative permittivity can be achieved with the probes. This is strongly related to the moisture content of the material, and some of the most promising models for calibrating the relative permittivity stem from the work of Lichtenecker [5], who showed that the relative permittivity of a medium with $N$ phases could be expressed by Equation (2).

$$
\varepsilon_{r}=\left(\sum_{i=1}^{N} \theta_{i} \varepsilon_{i}^{\alpha}\right)^{1 / \alpha}
$$

where $\theta_{\mathrm{i}}$ is the volume fraction of phase $i, \varepsilon_{\mathrm{i}}$ is the permittivity of phase $i$, and $\alpha$ is a constant between -1 and 1 .

Equation (2) can be rearranged to describe a porous material which has a solid matrix, and pores that may be filled with air or water. This has been utilised by Plagge [6] to give Equation (3).

$$
\varepsilon_{r}=\left[\theta \varepsilon_{w}{ }^{\alpha}+(1-\phi) \varepsilon_{s}{ }^{\alpha}+(\phi-\theta) \varepsilon_{g}{ }^{\alpha}\right]^{1 / \alpha}
$$

where $\varepsilon_{\mathrm{w}}$ is the relative permittivity of water, $\varepsilon_{\mathrm{g}}$ is the relative permittivity of air $(\approx 1), \varepsilon_{\mathrm{s}}$ is the relative permittivity of the solid matrix material, $\theta$ is the moisture content of the material and $\phi$ is the porosity of the material (open porosity for this application). To use this function for material measurement a value for $\alpha$ and $\varepsilon_{\mathrm{s}}$ must be determined from regression analysis of measurements of relative permittivity over a range of known moisture contents. Clearly some precalibration is required for accurate absolute moisture content measurements to be made.

For application to building materials TDR probes with two parallel waveguides have been developed. In soil sciences triple waveguide probes have become common, but for practical considerations associated with limitations of precise drilling of holes to accommodate waveguides a dual waveguide configuration has been adopted. Probes with waveguides of various diameters have been investigated and found to work in laboratory experiments. However, it has been observed that significant wear of drills and associated practical difficulties can occur when installation is attempted into some granular masonry materials such as sandstone. To help achieve realistic installation at a repeatable quality of contact, probes with waveguides of diameter of $4 \mathrm{~mm}$ were adopted. This increased diameter is easier to install using stiffer masonry drills, which are able to drill the sandstone in the dry state without overheating of the drill bit. The contact quality between waveguide and the building material is important, and affects the calibration between absolute moisture content and measured relative permittivity. 


\subsection{Dual Probe Heat Pulse Method}

The Dual Probe Heat Pulse Method uses a dual element probe which has a heating needle and a temperature sensing needle at a precise spacing. A short duration pulse of electrical energy (typically 10 seconds in duration) is applied to a wire within the heating needle. The temperature sensing needle, incorporates a thermocouple and measures the maximum temperature rise in the material at the set distance from the heating needle. This temperature rise, combined with the volumetric heat capacity of the dry material allows the moisture content to be determined. The technique is effectively insensitive to the presence of salts in the material being measured. If the physical properties of the substrate are not known, the relative changes in moisture content can still be accurately determined. The need for the substrate to cool back down to background levels after a reading means that at least one hour is needed between readings.

\subsection{Electrical techniques}

Electrical resistance or conductivity techniques are based on the principle that the conductivity of a material is dependent on its moisture content. In building investigations these techniques rely on the current being carried through the material by the ions in the pore solution and consequently are unsuitable for materials that exhibit electronic conduction. It follows that porosity is a major influence in the electrolytic conductivity of the material and hence calibration procedures are required for each material in order to relate the resistivity to the moisture content. Some materials have been widely investigated, most notably wood for which calibration tables are available for a wide range of species [7].

Basic electrical resistance or conductivity methods offer a simple low cost strategy for measuring moisture in building materials. The most basic systems operate by applying a d.c. voltage between two probes and measuring the resultant current. Although commercially available these devices offer very poor performance due to polarisation and electrode contact issues. Better performance is achievable using an a.c. excitation potential or current to overcome electrolytic polarisation of the probes. However, electrode contact problems may still be present.

The technique investigated in this study uses a constant a.c. current applied to the material under investigation by two probes. The resultant potential difference is measured with a lock-in amplifier and a data logger. Simple stainless steel anchor bolts are used as probes, which give good contact with the material.

\section{Calibration of test measurements}

All of the experimental techniques investigated are capable of producing a relative measurement of moisture content, by which the variation of moisture content with time can be assessed. However, to achieve accurate measurements of the absolute moisture content requires detailed calibration of probes in each 
material. In order to achieve this, a laboratory X-ray absorption method was used as the reference method.

The equipment (Figure 1) measures the absorption of X-rays which may be scanned in two dimensions over a sample [8]. Collimators placed in front of a Cadmium-Zinc-Telluride high-resolution detector can be used to define the spatial resolution of the X-ray system to less than one millimeter, with the positioning of the mechanical scanning system being repeatable to within $\pm 0.01 \mathrm{~mm}$. As a measurement at each point requires only a few seconds, transient changes can be evaluated in real time. A dedicated PC allows fullyautomated measurement, data collection and results analysis, with measurements being carried out in pre-determined patterns. Sequential measurement of a number of different samples mounted within the equipment is possible.

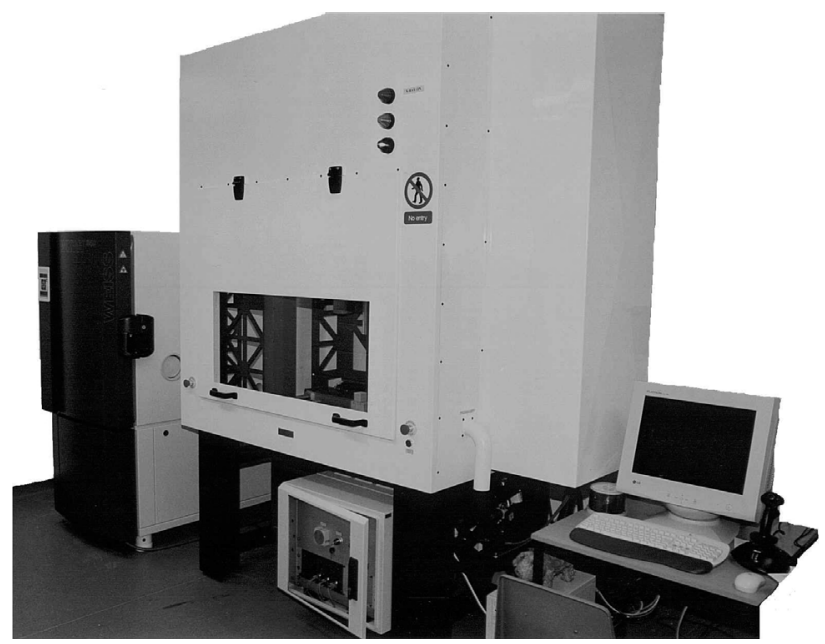

Figure 1: The X-ray facility at Glasgow Caledonian University.

The four vertical faces of a sample are covered with a water impermeable material, e.g. cling film or aluminium foil - it may then be assumed that the moisture distribution across the sample is reasonably uniform, since evaporation of moisture at the vertical faces is prevented. The sample is then mounted with its base on spacers in a container within the X-ray chamber.

A reference ('dry') scan is first made of the sample. The scans correspond with the location of the probes inserted for calibration. Water is then introduced to the base of the sample. Subsequent scans of the moist material are made in precisely the same positions as the reference scan. The Beer-Lambert law is applied to determine the moisture content as a function of the ratio of the X-ray absorption of the wet and dry material. For example, the volumetric moisture content may be expressed as follows: 


$$
\theta=\frac{\ln \left(\frac{I_{d r y}}{I_{w e t}}\right)}{d \mu_{w} \rho_{w}}
$$

where $\mathrm{I}_{\mathrm{dry}}$ is the transmitted X-ray intensity of dry sample, $\mathrm{I}_{\text {wet }}$ is the transmitted $\mathrm{X}$-ray intensity of wet sample, $\mathrm{d}$ is the thickness of the sample $(\mathrm{m}), \mu_{\mathrm{w}}$ is the mass attenuation coefficient of water and $\rho_{\mathrm{w}}$ is the density of water.

Introduction of a water source to the underside of the block of material in the $\mathrm{X}$-ray apparatus allows the wetting, and upon removal, drying, behaviour of the material to be observed. By undertaking simultaneous measurements of the $\mathrm{X}$-ray apparatus and the moisture sensor the calibration of sensor output against moisture content can be established through a long period of measurements. Full details of the calibration of the sensors has been described elsewhere [2, 3]. Unless explicit information is available to describe the material in detail, a calibration function is needed for each material to be measured. An example of a calibration function is shown in Figure 2, which relates the output from one of the improved electrical techniques to the moisture content within sandstone.

Polynomials, or other calibration functions, for example Equation (3) in the case of TDR, can be fitted to the calibration data to allow signal output to be interpreted in terms of absolute moisture content.

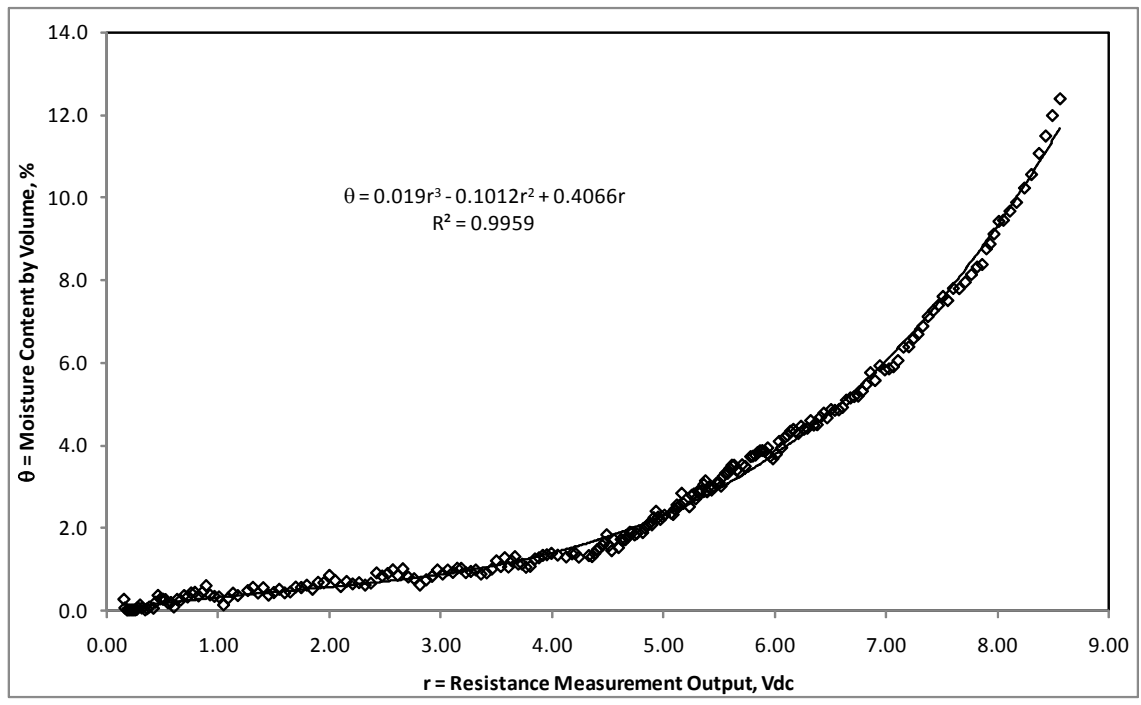

Figure 2: Calibration of electrical resistance measurement in comparison with moisture content measured by the X-ray absorption method. 


\section{Practical application}

The experience of using these techniques and application to a sandstone test wall is given below.

\subsection{Installation}

The original intention of the research had been to apply the techniques to a real wall on site. However, the logistics of keeping 3 separate systems installed for a period of time without the mass of cabling and logging equipment posing a nuisance to occupants was thought to be difficult and eventually a laboratory based practical application was adopted. A stone wall was available for testing in an environmental chamber which was a closely controlled environment to which the public had no access. In addition the authors were able to intervene by introducing effective flood conditions to the base of the wall to induce changes in the moisture content of the wall, and therefore allow the dynamic responses of the different sensors to be compared.

The intention of the installation was to mimic the conditions we would expect to see in a typical building under investigation, and as a consequence hardware for installing probes was limited to devices that could be easily carried and could be powered from mains electricity. Sophisticated workshop cutting techniques are unlikely to be ever available for drilling on site.

\subsubsection{Time domain reflectometry}

Installation of the probes into the test walls required the wall surface to be made flat using an angle grinder. An uneven stone wall surface will be the worst case for monitoring, however, no significant problems were encountered smoothing the surface. Drilling the twin holes for the waveguide used a template to ensure correct spacing was achieved for probe insertion. Holes were produced to the required tolerance to enable satisfactory fitting of the probe into the wall.

The decision to use larger $4 \mathrm{~mm}$ diameter waveguides for the TDR measurements made the installation of the probe far easier in sandstone. Time and attention to detail was needed to install the probe correctly, however the installed probe has been found to be responsive to the changing conditions of the test wall. Relative moisture contents can be inferred directly from the measured relative permittivity, and because the material has been tested in the laboratory against dedicated x-ray measurement equipment, a calibration curve can be used to assess absolute moisture content.

\subsubsection{Dual probe heat pulse method}

As with the TDR, the installation of the probes required that a flat surface be achieved with the sandstone test wall. The diameter of the probes used with this approach is just $1 \mathrm{~mm}$, necessary for the technique to work effectively. These small holes require a specialist drill and drill bit. A template was used to locate exactly the position of the holes. Problems were encountered trying to drill the dry sandstone with the drill head melting. Wetting of the material before and during drilling allowed holes to be successfully drilled, however, a number of 
drills were snapped in the process. The probes were installed into the material with a thermal paste used to eliminate any contact issues between the probe and the sandstone.

The installation of such fine probes into an abrasive material like sandstone is difficult. The need to add water as a coolant during drilling was unavoidable, but changes the moisture content and history. Although the authors successfully installed the probes into the sandstone wall, the technique would be better suited for use in materials that are easier to drill, e.g. autoclaved aerated concrete.

\subsubsection{Electrical techniques}

Installation of sensor points into the sandstone wall were achieved with the least difficulty of all three techniques. Pilot holes of $6 \mathrm{~mm}$ diameter were drilled into the sandstone and stainless steel expanding bolts were fixed into the material to achieve a good electrical contact with minimal risk of corrosion. Once in the electrodes are extremely difficult to remove so it is important to ensure that they are positioned correctly.

\subsection{Results from test walls}

The measurements of absolute moisture content determined using calibration functions and the output from the three sensor types on the sandstone wall are shown in Figure 3.

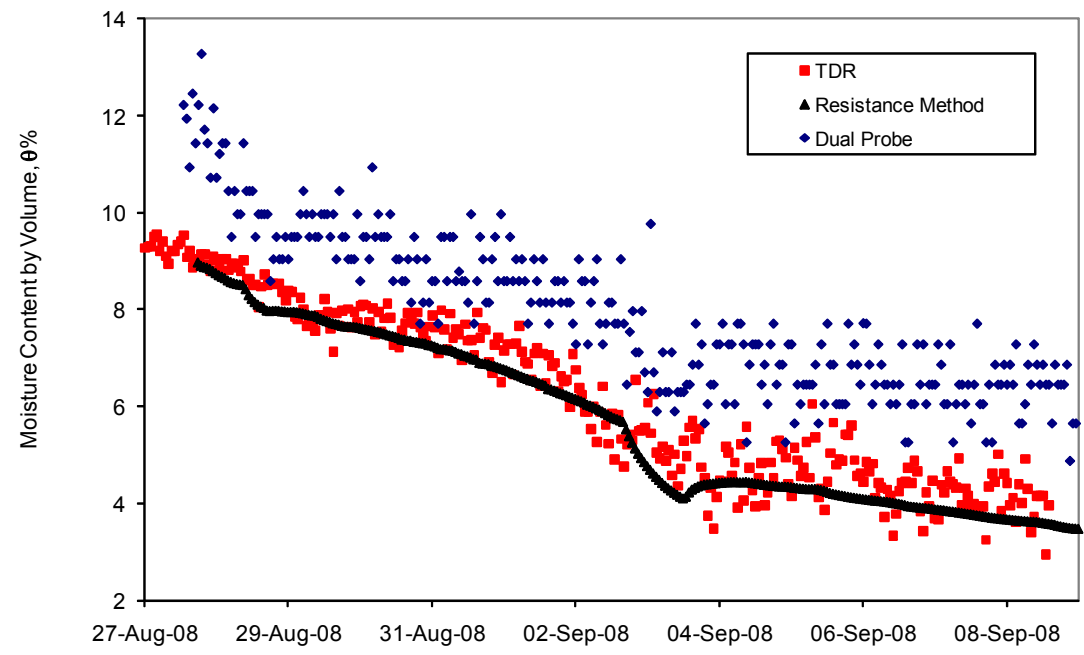

Figure 3: Absolute measurements of moisture content made for a stone test wall in an environmental chamber.

All three sensors show the same overall trend in the measurements of the absolute moisture content within the material. Some differences would be anticipated between the sensors as the volume of material measured differs significantly. For example, the TDR measures a volume of material up to $65 \mathrm{~mm}$ 
deep into the wall, whereas the dual probe technique measures the response much closer to the external surface of the wall. As the test wall is able to dry from all surfaces, there may be a difference between the moisture content within the core of the wall and that within the surface layers.

Each techniques has produced plausible measurements of the absolute moisture content, and so have the potential to be useful for the long term monitoring of the moisture behaviour of buildings or components.

\section{Conclusions}

Attention is needed when developing new moisture content measuring techniques for building materials to ensure that easy and consistent installation is possible. The quality of contact between measurement probes and the building fabric is important to achieve consistent measurements. Installation of probes on site with relatively limited mechanical tools requires both skill and a planned approach; it is therefore helpful if this practicality is considered at the design stage of the technique. Delicate and intricate probes require significant preparation to be installed into a material, and as found with the dual probe, some materials are extremely difficult to work with.

As the majority of existing laboratory based techniques with high accuracy are not portable, the development of research tools for site use is an important challenge for building physics. If research techniques are ever to become a mainstream measurement option for building professionals, they need to be able to be installed to a high standard with minimum effort.

All three techniques have shown promise in monitoring the test wall, although as noted above, the dual probe took an excessive amount of time to install in sandstone, its application may be better suited to other substrates.

\section{References}

[1] Eidmann G, Savelsberg R, Blümler P, Blümich B. The NMR mouse, a mobile universal surface explorer. Journal of Magnetic Resonance Series A., 122: 104-109, 1996.

[2] Ye Z., Titrovic M., Davies M., Baker PH., Phillipson M., Galbraith GH., McLean RC. The optimization of a thermal dual probe instrument for the measurement of moisture content of building envelopes. Building Serv. Eng. Res. Technol., 28 (4), pp. 317-327, 2007.

[3] Phillipson MC, Baker PH, Davies M, Ye Z, Galbraith GH, McLean RC. Suitability of time domain reflectometry for monitoring moisture in building materials. Building Serv. Eng. Res. Technol., 29 (3), pp. 261-272, 2008.

[4] Phillipson MC, Baker PH, Davies M, Ye Z, McNaughtan A, Galbraith GH, McLean RC. Moisture measurement in building materials: an overview of current methods and new approaches. Building Serv. Eng. Res. Technol., 28, 4, pp. 303-316, 2007.

[5] Lichtenecker K. Die dielektrizitatskonstane naturlicher und kunstlicher mischkorper. Phys. Z. 27, 115-58, 1926. 
[6] Plagge R. Bestimmung von Materialfeuchte und Salzgehalt in kapillar porosen Materialien mit TDR. Koloquium mit Workshop Innovative feuchtemessung in Forschung und praxis. Karlsruhe, 2003.

[7] Hoadley R B., Understanding Wood, 2000, The Taunton Press.

[8] Baker PH, Bailly D, Campbell M, Galbraith GH, McLean RC, Poffa N, Sanders $\mathrm{CH}$. The application of X-ray absorption to building moisture transport studies. Measurement. 2007; 40: 951-959. 